\title{
La politique culturelle de l'an II : les infortunes de la propagande révolutionnaire au théâtre
}

\section{Martin Nadeau}

\section{(2) OpenEdition \\ 1 Journals}

\section{Édition électronique}

URL : https://journals.openedition.org/ahrf/559

DOI : 10.4000/ahrf.559

ISSN : 1952-403X

Éditeur :

Armand Colin, Société des études robespierristes

\section{Édition imprimée}

Date de publication : 1 mars 2002

Pagination : $57-74$

ISSN : 0003-4436

\section{Référence électronique}

Martin Nadeau, "La politique culturelle de l'an II : les infortunes de la propagande révolutionnaire au théâtre ", Annales historiques de la Révolution française [En ligne], 327 | janvier-mars 2002, mis en ligne le 19 mars 2008, consulté le 23 avril 2022. URL : http://journals.openedition.org/ahrf/559 ; DOI :

https://doi.org/10.4000/ahrf.559 


\title{
LA POLITIQUE CULTURELLE DE L'AN II: LES INFORTUNES DE LA PROPAGANDE RÉVOLUTIONNAIRE AU THÉÂTRE
}

\author{
MARTIN NADEAU
}

\begin{abstract}
La conceptualisation de la Révolution française en termes de pratiques nouvelles qui surinvestissent la politique de significations symboliques a sollicité cette étude du rôle du théâtre sur la scène politique révolutionnaire. Le théâtre, une pratique culturelle nettement dominante à cette époque, a attiré à juste titre l'attention des diverses instances de l'autorité politique. S'y observe un mouvement de pendule entre liberté, avec le décret du 13 janvier 1791, et surveillance étroite, avec celui du 2 août 1793. La confrontation des comptes rendus de représentations théâtrales avec les volontés parfois contradictoires de l'Assemblée nationale, de la municipalité de Paris, de la Commune insurrectionnelle, du Comité de salut public et du Comité d'instruction publique, exprime des enjeux inhérents au débat sur la médiation de la volonté générale. En tant que pratique culturelle publique, le théâtre instaure un régime de discours complexe, très instable, ou les acteurs et les actrices, mais aussi le public, exercent un rôle de coauteur dans la configuration du sens d'une représentation théâtrale. II s'agit d'une pratique culturelle qui s'avère être ainsi très difficile à contrôler du point de vue des autorités politiques et ce aussi bien à l'égard de la propagande, qu'à celui de la censure.
\end{abstract}

Mots clés : Théâtre ; Révolution ; représentation et volonté générale.

Il est possible de dater l'intention du Comité d'instruction publique de faire des théâtres une «école du peuple » en mai 1792, à partir d'une lettre d'un de ses membres, Gilbert Romme, qui répond favorablement à une proposition de l'auteur dramatique Jean-François Cailhava de l'Estandoux de former une école pour enseigner l'art de la comédie, dans l'esprit que le théâtre doit être lui-même une école (1). Avant d'analyser la mise en place

(1) Josiane BOULAD-AYOUB et Michel GRENON (éds), Édition nouvelle et augmentée des procès-verbaux du Comité d'instruction publique de l'Assemblée législative et de la Convention publiés et annotés par James Guillaume, 9 volumes, préface de Michel Vovelle, Paris, l'Harmattan, 1997 (Paris, 1889). Désormais AYOUB et GRENON, CIP. Voir AYOUB et GRENON, CIP, volume II, Assemblée législative, « Pièces annexes », p. 191. 
de ce processus d'instrumentalisation du théâtre, dans lequel le Comité d'instruction publique a joué un rôle de premier plan, il est opportun de considérer quelques comptes rendus de la vie théâtrale du début de la Révolution, qui permettront d'appréhender la pertinence et la portée réelle de ces mesures ultérieures.

Les auteurs de la Chronique de Paris, notamment Aubin-Louis Millin de Grandmaison, sont très éloquents à cet égard (2). Dans un premier temps ce chroniqueur des spectacles, en mars 1791, reconnaît l'importance du décret sur la liberté des théâtres (13 janvier 1791) qui abolit la censure dite d'Ancien Régime, ainsi que le monopole des trois théâtres privilégiés. Ce décret entraîne la prolifération des salles de spectacles à Paris : il y en aura jusqu'à 36 en 1792 (3). Millin de Grandmaison remarque que la représentation de pièces qui cèdent au goût du jour, dans ce cas-ci l'anticléricalisme, devient superficielle : «On a commencé par les Dominicains, les Bénédictins; sont parus enfin les Capucins : cependant aucun des ouvrages qui n'avoient pour but que la représentation du costume monacal, n'a réellement réussi. [...] Ces moyens factices ne séduiront plus personne» (4). Il poursuit sa réflexion en déplorant aussi la redondance dans laquelle tombent les pièces politiques qui cherchent à profiter de la vogue patriotique. Ce critique insiste sur la nécessité du retour à la spécificité de la pratique théâtrale chez les « grands écrivains », c'est-à-dire la représentation de pièces qui cherchent plus la «connoissance du cœur humain » que la conformité au goût du jour. En septembre 1791, nous retrouvons de nouveau cette mise en garde contre les pièces de circonstance :

« Nous saisissons cette occasion d'observer qu'à la longue rien ne deviendra plus fastidieux que ces pièces de circonstance. Nous invitons les auteurs

(2) La Chronique de Paris, fondée le 24 août 1789, est le second quotidien de la capitale avec le Journal de Paris, qui lui existe depuis 1777. Il s'agit d'un journal qui a pu être qualifié d'obédience « girondine », puisque Condorcet y a rédigé des comptes rendus des débats de l'Assemblée nationale. Censuré en juin 1793, ce quotidien, qui n'est pas a priori un journal littéraire, constitue, de par la richesse et la profusion des comptes rendus des spectacles de Millin de Grandmaison, une source précieuse pour l'étude de la vie théâtrale parisienne entre 1789 et 1793 . Sur les rapports entre la critique théâtrale et la politisation de la sphère publique littéraire voir Michel BIARD, « De la critique théâtrale, ou la conquête de l'opinion », Annales historiques de la Révolution française, 1995, n 4, pp. 529-538, et aussi Gregory S. BROWN, «Le Fuel de Méricourt and the Journal des Théatres: Theatre Criticism and the Politics of Culture in PreRevolutionary France », French History, 1995, n 1, pp. 1-26.

(3) La Chronique de Paris, 79 (20 mars 1791) : 314. Pour la description de toutes ces nouvelles salles de spectacle on peut se référer à Giuseppe RADICCHIO et Michèle SAJOUS D'ORIA, Les Théâtres de Paris pendant la Révolution, traduit de l'italien par Laura Casati et Françoise Lenoir, Paris, Bibliothèque de la ville de Paris, 1990, et aussi à André TISSIER, Les Spectacles à Paris pendant la Révolution. Répertoire analytique, chronologique et bibliographique 1789-1792, Genève, Librairie Droz, 1992. Emmet Kennedy, MarieLaurence Netter, John P. McGregor, Mark V. Olsen, dans Theatre, Opera and Audiences in Revolutionary Paris. Analysis and Repertory, Wesport, Greenwood Press, 1996, ont poursuivi le travail de lissier pour l'ensemble de la période révolutionnaire.

(4) La Chronique de Paris, 79 (20 mars 1791) : 314. Rappelons que l'abolition des vœux monastiques date du 13 février 1790, que la Constitution civile du clergé a été votée le 12 juillet 1790 et qu'un premier bref pontifical a condamné cette dernière le 10 mars 1791 . 
dramatiques à revenir aux bons genres, $\&$ au bon goût, $\&$ à ne pas prendre de la niaiserie pour de la naïveté, du bas pour du naturel, des calembours pour de l'esprit, \& du jargon pour du style. » (5)

Certes c'est au nom du goût que cet auteur parle, mais aussi d'une certaine manière au nom de l'indépendance du théâtre : il poursuit, quelques jours plus tard, en réitérant cette idée, à propos d'une de ces pièces de circonstance intitulée Artémidor, que le patriotisme ne suffit pas pour faire le succès d'un ouvrage :

«Dernièrement on a donné, au théâtre du Marais, une tragédie en trois actes, intitulée Artémidor. La scène se passe à Syracuse, \& l'action est une allégorie de notre révolution. Si le patriotisme suffisoit pour faire le succès d'un ouvrage, celui-ci en mériteroit un brillant ; mais comme il importe au maintien \& à la conservation du goût que toutes les règles des arts ne soient pas violées et confondues, nous sommes forcés de dire que de longues déclamations sans intérêt, dans lesquelles on répète trois cents fois les mots citoyens \& liberté, finiront par anéantir l'art dramatique en France. Il est tems d'arrêter ce débordement d'ouvrages de circonstance. » (6)

Avec les mesures de l'an II prises par le Comité de salut public, qui s'imposera sous la Terreur sur son homologue de l'instruction publique, c'est exactement le contraire qui se produira : ces ouvrages de circonstance "déborderont".

Dans le contexte politique tumultueux du début de 1793, la représentation de deux pièces de théâtre, soit L'Ami des lois de Jean-Louis Laya et La Chaste Suzanne des auteurs Barré, Radet et Desfontaines, peut être considérée comme le catalyseur d'un important processus d'instrumentalisation des théâtres (7). Un processus culminant avec le décret du 2 août 1793 sur la surveillance des spectacles, qui inversera le décret de 1791 sur la liberté des théâtres. Aucune pièce sans doute sous la Révolution n'a suscité d'aussi graves incidents que L'Ami des lois. Les représentations qu'en donna le Théâtre de la Nation ont entraîné, à terme, la fermeture de cette salle et l'arrestation de ses acteurs (8). Cette comédie dite souvent «d'inspiration girondine », car elle met en scène Robespierre sous les traits de Nomophage et Marat sous ceux de Duricrâne - les applications qu'a pu effectuer le public sont allées semble-t-il de soi -, a suscité l'indignation compréhensible

(5) Id., 268 (26 septembre 1791): 1085.

(6) $I d, 275$ (2 octobre 1791) : 1109.

(7) Voir sur le contexte entourant la représentation de ces deux pièces à Paris en 1793, Suzanne JEAN-BÉRARD, « La Crise du théâtre en 1793 », Dix-Huitième Siècle, 1989, n 21, pp. 411-422.

(8) Voir Jacques TRUCHET (éd.), Théâtre du XVIIIe» siècle, 2 volumes, Paris, Gallimard, coll. « La Pléiade », 1972, notice de L'Ami des lois, volume 2, p. 1543. Voir aussi à ce sujet Frederick W. J. HEMMINGS, Theatre and State in France, 1760-1905, New York, Cambridge University Press, 1994, pp. 79-82. Rappelons que le Théâtre de la Nation à cette époque correspond à l'aile noire ou conservatrice de la ComédieFrançaise, dont une partie des comédiens en avril 1791, sous l'impulsion des convictions révolutionnaires de l'acteur Talma, s'est établie rue de Richelieu dans ce que l'on nommera le Théâtre de la République ou l'aile rouge de la ci-devant Comédie-Française. 
des proches de Robespierre, comme Chaumette, procureur de la Commune de Paris, et Hébert son substitut. Le très officiel Moniteur Universel témoigne du succès de cette pièce auprès du public le 3 janvier 1793, alors que se déroule le procès du roi : «Laya a été demandé ; il a paru, et a reçu les plus vifs applaudissements » (9).

Laya en portant sa pièce devant la Convention - majoritairement « girondine » à l'époque - a suscité un débat houleux le 10 janvier 1793 , mais encore aucune prise de position légale. C'est en vain d'abord que la Commune de Paris a tenté de faire suspendre les représentations le lendemain. Un procès-verbal du Comité d'instruction publique daté du 11 février 1793 témoigne que cette question a été renvoyée de la Convention au Comité, qui déclare alors que «la Convention nationale ne devrait fixer son attention sur aucun ouvrage, à moins qu'il ne fût relatif à l'objet de ses travaux» (10). Mais c'est bien la Commune qui finit par l'emporter, c'est-àdire interdire la représentation de L'Ami des lois, sinon en droit, du moins par la force et par l'intimidation (11). Un problème mis en scène précisément par l'intrigue de cette pièce.

Le droit de censure appartient légalement, selon le décret de janvier 1791, à la municipalité. C'est ce que rappellera le Comité d'instruction publique, après avoir été sollicité une nouvelle fois à cette époque par les auteurs dramatiques Barré, Radet et Desfontaines, qui ont écrit une pétition afin de justifier le «civisme» de La Chaste Suzanne. La représentation de cette pièce a été troublée parce que des spectateurs ont refusé qu'un bis, demandé pour les couplets suivants du Vaudeville final de la pièce, soit exécuté :

« Affecter candeur et tendresse,

Du plus offrant que l'amour presse

Recevoir argent et présent,

C'est ce que l'on fait à présent.

Refuser plaisir et richesse,

Pour conserver gloire et sagesse

De la mort souffrir le tourment,

Oh ! c'est de l'Ancien Testament. »

Les auteurs, dans leur pétition, soulignent que « [c]omme il n'est question dans ce couplet que d'une femme qui vend son honneur et d'une autre femme qui brave la mort pour le conserver, toute interprétation maligne est fausse et ridicule » (12).

(9) La Gazette nationale ou Le Moniteur universel, 4 (4 janvier 1793) : 30.

(10) AYOUB et GRENON, CIP, volume III, Convention, tome 1, p. 243.

(11) TRUCHET, notice de L'Ami des lois, p. 1544. Voir aussi Pierre FRANTZ, « Les tréteaux de la Révolution », dans Jacqueline DE JOMARON (dir.), Le Théâtre en France, préface d'Ariane Mnouchkine, Paris, Armand Colin, coll. « La Pochothèque », 1992, p. 514.

(12) AYOUB et GRENON, CIP, volume III, Convention, tome 1, annexe C, «Pétition des citoyens Barré, Radet et Desfontaines, justifiant le civisme de La Chaste Suzanne», datée du 29 janvier 1793, p. 246. 
Cette «interprétation maligne» de la part de certains spectateurs témoigne de l'importance des applications conflictuelles opérées par le public. Ce dernier est composé de spectateurs issus d'origines sociales très diversifiées, dont les clivages politiques se transposent dans l'élaboration du sens d'une pièce (13). Les vers du premier couplet «Recevoir argent et présent | C'est ce que l'on fait à présent» ont pu en effet être perçus comme une critique du Nouveau Régime et applaudis en conséquence par des spectateurs qui, vraisemblablement, regrettent une certaine éthique de l'honneur attachée à l'Ancien. D'où les problèmes particuliers que pose la question de la censure du théâtre. C'est à propos de cette pièce jouée, je le rappelle encore, à l'époque du procès du roi, que Pierre Frantz a très justement affirmé que les représentations les plus significatives, politiquement, ne furent pas toujours celles que le titre de la pièce, ou leur contenu imprimé, laisserait présager (14). Frantz, plutôt que de faire référence au couplet dont parlent les auteurs dans leur pétition, dit cependant que c'est la réplique «Vous avez été ses dénonciateurs, vous ne sauriez être ses juges », appliquée bien sûr au contexte politique, qui a déclenché le tumulte.

Le 2 juillet 1793, explicitement pour la première fois dans les rapports du Comité d'instruction publique, est fait mention des spectacles du point de vue de leur instrumentalisation. À la toute fin d'une «motion d'ordre sur la discussion de l'instruction publique » par J.-M. Coupé, figure un article sur les spectacles, assez vague dans son objet, mais qui souligne néanmoins :

« $[\mathrm{Qu}$ '] ils sont devenus la partie la plus puissante de l'instruction publique. [...] Que la poésie consacre les traits héroïques et les grands exemples de nos concitoyens; que toute l'Europe vienne admirer le génie qui sait faire et célébrer tant de merveilles, et que ces scènes enchantées soient répétées dans toutes nos villes et sur les places de nos cantons, au milieu des exercices militaires et des accents de la musique. » (15)

Coupé souligne ici un effet enchanteur des spectacles qui semblent être assimilés aux fêtes nationales, dont la nécessité est établie dans les différents projets d'instruction publique, mais non sans susciter des débats parmi les membres de ce comité. La position de Condorcet est bien connue : il s'oppose catégoriquement à ces formes de « séduction sociale » que sont les fêtes nationales (16). En appendice à cette motion d'ordre de Coupé, est reproduit

(13) Sur la composition sociale du public des théâtres au XVIII siècle et sous la Révolution, voir Jeffrey S. RAVEL, The Contested Parterre. Public Theater and French Political Culture 1680-1791, Ithaca, Cornell University Press, 1999. Cet historien souligne l'extrême hétérogénéité du public des parterres. Il a établi qu'on y retrouve côte à côte des financiers, des officiers, des apprentis, des étudiants. Ravel parle ainsi de « fissures » au sein du public des parterres qui révèlent la fragilité des catégories sociales utilisées parfois pour distinguer un public riche ou d'élite d'un public populaire, dont le comportement serait déterminé par leurs mentalités respectives. Voir notamment p. 19.

(14) Pierre FRANTZ, «Les tréteaux de la Révolution », op. cit., p. 512.

(15) AYOUB et GRENON, CIP, volume III, Convention, tome 1, p. 408.

(16) Id., volume I, introduction générale, p. 22. 
un article de Condorcet publié dans La Chronique du mois de janvier 1793 et intitulé «Sur la nécessité de l'instruction publique ». Il s'agit d'une nécessité pour Condorcet, car il redoute précisément «l'hypocrisie des tyrans, qui savent prendre le masque de la popularité, ou celui de la justice ». Et il ajoute que ceux qui disent «que le peuple en sait assez, s'il sait vouloir être libre », avouent en fait «qu'on veut le tromper pour s'en rendre maître ». Condorcet dénonce toutes les formes de «séduction sociale », car «c'est le dégrader [le peuple] sous la vaine apparence d'un respect perfide » (17).

On peut imaginer à quel point les idées de Condorcet à ce sujet seront ignorées à la suite des journées des 31 mai, 1er et 2 juin 1793, qui évincent les Girondins, auxquels son nom reste à tort ou à raison attaché, de l'enceinte de la Convention nationale. Condorcet sera dénoncé devant l'Assemblée le 8 juillet 1793 pour avoir manifesté son indignation devant la Constitution de 1793 et c'est dans la clandestinité qu'il élaborera désormais ses réflexions. C'est donc une Convention nationale dite montagnarde qui a décrété la loi du 2 août 1793 sur la surveillance des spectacles. Le procèsverbal de la séance du 2 août 1793 à la Convention décrit l'état d'esprit dans lequel est votée cette loi, à l'initiative non pas du Comité d'instruction publique, mais bien du Comité de salut public : «Le Comité de salut public, voyant s'approcher la fête du 10 août, à laquelle doivent assister les députés des départements, chargés de présenter l'acceptation de la constitution, et désirant former de plus en plus, chez les Français, le caractère et les sentiments républicains, propose une loi de règlement sur les spectacles ». Un premier article inscrit dans la loi l'instrumentalisation des théâtres à des fins de propagande ou de persuasion politique :

« À compter du 6 de ce mois, et jusqu'au $1^{\text {er }}$ septembre prochain, seront représentées trois fois la semaine, sur les théâtres de Paris qui seront désignés par la municipalité, les tragédies de Brutus [Voltaire], Guillaume Tell [Lemierre], Caïus Gracchus [Chénier], et autres pièces dramatiques qui retracent les glorieux événements de la Révolution, et les vertus des défenseurs de la liberté; une de ces représentations sera donnée chaque semaine aux frais de la République. » (18)

Le second article, quant à lui, rétablit proprement la censure des théâtres, mais sans faire allusion au décret de 1791 : «Tout théâtre sur lequel seraient représentées des pièces tendant à dépraver l'esprit public, et à réveiller la honteuse superstition de la royauté, sera fermé, et les directeurs arrêtés et punis selon la rigueur des lois». Enfin, le troisième et dernier article stipule que l'application de ce décret voté par la Convention est confiée à nouveau à la municipalité de Paris (19).

(17) Id., volume III, Convention, tome 1, p. 469.

(18) Id., volume IV, Convention, tome 2, annexe B, p. 491.

(19) Id. 
Ce décret sur les spectacles n'est évidemment qu'un aspect de la politique d'instrumentalisation des arts et de la culture en général préconisée par le gouvernement montagnard un mois avant l'établissement de la Terreur à l'ordre du jour, le 5 septembre. La figure emblématique de cette politique d'instrumentalisation des arts est bien entendu le peintre David, que nous pourrions qualifier d'impresario de la Révolution. Nous pourrions même le considérer comme le tout premier ministre de la culture dans l'histoire occidentale, car il incarne véritablement cette ambition aux multiples avatars de «former un nouveau peuple », se traduisant notamment par l'organisation des fêtes nationales (20). C'est précisément dans le contexte de la fête nationale du 10 août, en 1793, que le décret sur la surveillance des spectacles est voté. Les procès-verbaux du Comité d'instruction publique permettent de retracer le programme de cette fête, orchestrée dans les moindres détails par David; une fête qui doit d'ailleurs se terminer par la représentation de pantomimes relatant les principaux événements de la Révolution sur un «vaste théâtre » (21).

Pour les députés de la Convention, l'utilité du décret du 2 août 1793 du point de vue de la censure est tout aussi appréciable. La représentation de la pièce Paméla de François de Neufchâteau (d'après le célèbre roman de Samuel Richardson) au Théâtre de la Nation, au début de septembre 1793, a donné lieu à sa première application. Le Moniteur rapporte les propos de Barère à ce sujet lors d'une séance de la Convention le 3 septembre 1793 :

«Le Théâtre de la Nation, qui n'était rien moins que national, a été fermé. Cette disposition est une suite du décret du 2 août 1793, portant qu'il ne serait joué sur les théâtres de la République que des pièces propres à animer le civisme des citoyens. La pièce de Paméla, comme celle de L'Ami des lois, a fait époque sur la tranquillité publique. On y voyait non la vertu récompensée, mais la noblesse; les aristocrates, les modérés, les Feuillants se réunissaient pour applaudir les maximes proférées par des mylords. » (22)

Après avoir souligné que le principal vice de la pièce en question est le «modérantisme» et qu'en conséquence non seulement le Comité de salut

(20) Voir David LOYD DOWD, Pageant-Master of the Republic. Jacques-Louis David and the French Revolution, Lincoln, University of Nebraska Studies, 1948. Voir aussi Mona OZOUF, La Fête révolutionnaire (1789-1799), Paris, Gallimard, 1976, et Serge BIANCH, La Révolution culturelle de l'an II. Élites et peuple 1789-1799, Paris, Aubier Montaigne, 1982. Dowd insiste davantage sur le rôle des arts graphiques, les gravures et les peintures, tandis qu'Ozouf, évidemment, appréhende les fêtes révolutionnaires. Dans les deux cas, peu d'attention est accordée au théâtre. Bianchi consacre en revanche quelques pages intéressantes à cette pratique : voir la section « le peuple au théâtre» pp. 220-225. On trouvera des considérations importantes sur David dans Claudette HOULD, Les Beaux-arts et la politique durant la Révolution française : problème de la propagande, 1789-1794, Montréal, Presses de l'Université de Montréal, 1971, et aussi dans James A. LEITH, The Idea of Art as Propaganda in France 1750-1799 : a Study in the History of Idea, Toronto, University of Toronto Press, 1965.

(21) AYOUB et GRENON, CIP, volume IV, Convention, tome 2, « Rapport et décret sur la fête de la réunion républicaine du 10 août, présenté au nom du Comité d'instruction publique par David », p. 59.

(22) Id., annexe B, pp. 490-491. 
public a fait arrêter les acteurs et les actrices du Théâtre de la Nation, mais aussi l'auteur lui-même, Barère nous livre sa profession de foi sur l'utilité des théâtres, partagée semble-t-il par les autres députés de la Convention : « Si cette mesure paraissait trop rigoureuse à quelqu'un, je lui dirais : Les théâtres sont les écoles primaires des hommes éclairés et un supplément de l'éducation publique. L'assemblée applaudit à cette mesure, et la confirme » (23).

Un procès-verbal de la séance du 24 octobre 1793 du Comité d'instruction publique permet cependant de constater que, sur le terrain, dans les théâtres de Paris, les témoins et les agents de l'application de cette mesure officielle sont d'un tout autre avis. Un « citoyen administrateur de la police de Paris» a demandé en effet une audience au Comité d'instruction publique afin de signaler que les «opéras donnés gratuitement au public ne ravivent point l'esprit public ». Bien au contraire, «ils occasionnent beaucoup de disputes $\gg(24)$.

Si le Comité d'instruction publique semble s'accorder pour «laisser subsister les choses telles qu'elles sont » (25), La Feuille de Salut public, un organe officiel du gouvernement qui a remplacé La Chronique de Paris désormais censurée, offre, le 20 novembre 1793, des réflexions sur le théâtre qui s'y opposent et réclament la proscription des genres théâtraux de l'Ancien Régime. Le temps est venu d'un «gouvernement énergique » qui rétablira l'ordre dans les salles de spectacle si souvent aux prises avec la diversité du politique. Désormais, il faut que «toutes les pièces soient patriotiques »; que soient proscrits le «royalisme impudent » et l'«astucieux modérantisme »(26). Il ne suffit pas de représenter des pièces patriotiques, comme Le Jugement dernier des rois de Sylvain Maréchal, écrit au lendemain de l'exécution de Marie-Antoinette le 16 octobre 1793, mais il faut aussi que les genres du drame et des «farces scandaleuses » du théâtre à l'italienne soient censurés et proscrits :

«Proscrivons sans pitié ces drames insignifians, qui n'ont d'autre mérite que celui du style, et dont le but ne fut jamais d'inspirer un sentiment vertueux. Repoussons avec mépris ces farces scandaleuses qui outragent à la fois le bon goût et les mœurs. Ne souffrons plus que des petits-maîtres, des chevaliers, des marquis, avec leurs habits tout brillants de paillettes, distribuent des soufflets et des coups de bâton à ceux qui les servent, et qui semblent quelquefois honorés de ces singulières faveurs. Il n'est plus de valets, parce qu'il n'y a plus de maîtres. C'est déshonorer l'homme que de le représenter dans un tel état d'asservissement. »

(23) Id.

(24) Id., « séance du 24 octobre 1793 », p. 489.

(25) Id.

(26) Extrait de La Feuille de salut public, cité dans Le Journal des Spectacles, 141 (20 novembre 1793) : 1119-20. 
Mais La Feuille de salut public indique que ces genres, malgré tout, obtiennent un certain succès auprès du public :

«Si les spectateurs qui applaudissent par habitude à ces gentilesses de l'Ancien Régime, avoient le courage de les couvrir des huées qu'elles méritent, bientôt nos théâtres seroient la véritable école des mœurs. Le tableau des vertus sociales, le triomphe de la raison sur les préjugés, l'amour de la patrie, la passion de la liberté, la haine des tyrans, la légèreté et la frivolité vouées au mépris, quelle mine féconde pour l'écrivain patriote ! »

Enfin, en parlant du devoir chez les législateurs de «diriger l'esprit public », La Feuille de salut public réclame l'utilisation du théâtre, «un mobile puissant », au même titre que les fêtes nationales, afin d'accélérer la marche révolutionnaire :

«La marche révolutionnaire déjà devenue plus rapide, depuis que l'intrigue et la perfidie n'entravent plus les opérations de nos législateurs, le seroit encore davantage si, comme dans les fêtes nationales, le citoyen voyoit tous les jours, sur le théâtre, les brillantes époques de la révolution retracées avec une énergie patriotique; si les spectacles qui ont tant d'influence sur les moeurs publiques lui offraient les traits d'héroïsme qu'enfante l'amour de la liberté et la touchante image des vertus républicaines. Législateurs, magistrats du peuple, vous qui devez diriger l'esprit public, je vous présente un mobile puissant, sachez l'employer ! » (27)

La Convention a donc cherché, depuis le décret du 2 août 1793, à employer ce «mobile puissant». Mais le 15 novembre 1793, considérant que «les spectacles, vu leur influence dans l'éducation publique, ne peuvent être livrés à des spéculations particulières et privées », elle s'interroge encore sur le moyen de les « rendre nationaux et utiles à la liberté et au bonheur de la République » (28). C'est à la fin de cette séance de la Convention que Fabre d'Églantine, lui-même auteur dramatique, parle du théâtre comme d'une «espèce de sacerdoce qui s'exerce sur la pensée » (29).

Nous retrouvons dans les Archives parlementaires une intervention de Lombard-Lachaux, en janvier 1794, qui, au nom du Comité des finances de la Convention, permet d'étudier l'application concrète du décret du 2 août 1793. C'est lui qui a dû fixer les frais de la République pour que le théâtre ne soit plus aux mains des «spéculations particulières et privées » :

«En exécution de votre décret du 2 août (vieux style), les vingt spectacles de Paris ont correspondu à vos vues en s'empressant, les uns de donner d'abord les trois pièces désignées, et les autres celles qui étoient les plus propres à former l'esprit public que l'on avoit tant cherché à pervertir. Votre

(28) AYOUB et GRENON, CIP, volume IV, Convention, tome 2, « extrait du procès-verbal de la Convention du 25 brumaire », p. 618.

(29) Id., p. 620. 
comité des finances a été chargé de fixer l'indemnité qui étoit due à ces divers théâtres, lesquels ont donné chacun quatre représentations, comme vous l'aviez décrété. » (30)

Lombard-Lachaux montre ensuite le calcul que le Comité des finances a effectué afin de fixer ces indemnités. Le minimum du produit de quatre représentations correspondant à 28932 livres est fixé à partir de la plus basse saison des théâtres parisiens, soit le mois de juillet où les fortes chaleurs invitent les spectateurs à ne pas se rassembler dans des salles de spectacles mal aérées. Le maximum, correspondant à la haute saison, soit les mois d'hiver où inversement la chaleur des salles de spectacle constitue un attrait, est fixé à 188800 livres. Or si le mois d'août au cours duquel s'est déroulé ce cycle de représentations aux frais de la République - le décret précise bien du 6 août au $1^{\text {er }}$ septembre -, correspond davantage à la basse saison, ce qui implique que l'indemnité devrait être fixée en fonction des revenus anticipés normalement à ce moment, soit 28932 livres, LombardLachaux remarque cependant que le mois d'août 1793 a coïncidé avec «l'affluence des députés des cantons qu'avoit attirés à Paris la cérémonie du 10 août». Ainsi puisque l'affluence à Paris en ce mois d'août 1793 a été importante, les théâtres, qui auraient dû profiter financièrement de celle-ci, seront donc dédommagés en conséquence : une moyenne proportionnelle entre ces deux extrêmes du minimum et du maximum est proposée, «à peu près celle de cent mille livres, formant l'indemnité qu'une rigoureuse justice vous demande pour les vingt spectacles de Paris » (31).

Afin de justifier cette indemnité, Lombard-Lachaux a fait état également d'un saccage occasionné dans plusieurs théâtres qu'il interprète comme étant le résultat d'une affluence considérable :

«Nous avons aussi cru devoir prendre en considération les dégâts et dommages occasionnés dans plusieurs théâtres, par l'immense concours des spectateurs qui ont fracturé, sali, enlevé une foule d'objets qu'il a fallu rétablir à grands frais, comme cela est constaté par un état certifié du ministre de l'Intérieur, de même que les nouvelles décorations, l'augmentation des soldats comparans, et celle de la garde que nécessitoit une réunion d'individus aussi conséquente. » (32)

Lombard-Lachaux ne déplore et n'explique pas autrement ce saccage et il passe aussitôt à l'établissement de la part de ces 100000 livres d'indemnité qui revient à chacun des vingt théâtres concernés (33).

Le projet de décret sur l'organisation de l'instruction publique proposé par Gabriel Bouquier forme une autre étape importante dans cette histoire

(30) Archives parlementaires, tome 83, p. 560 (séance du 22 janvier 1794).

(31) Id.

(32) Id.

(33) Id., p. 561. 
de l'instrumentalisation du théâtre. Par le décret du 29 frimaire an II (19 décembre 1793), ce projet devient la première loi scolaire de la République ; mais tout sera à recommencer avec la chute de Robespierre. Quoi qu'il en soit, le théâtre a joué un rôle de premier plan dans le programme d'instruction publique élaboré par Bouquier. Ce dernier est luimême auteur de théâtre, comme l'indique le procès-verbal du Comité d'instruction publique du 20 octobre 1793 relatant la lecture d'une «SansCulottide» écrite par lui et s'intitulant La Réunion $d u 10$ août ou l'Inauguration de la République française (34). Nous retrouvons un procèsverbal de la Convention à la même époque où est mentionné très significativement que «L'Opéra-Comique esquiva la charge qui lui incombait» (35), c'est-à-dire que le ci-devant Théâtre-Italien a refusé de représenter cette pièce. A-t-elle été jugée mauvaise ? Une intervention à la Convention en février 1794 permet peut-être de répondre par l'affirmative. À l'annonce par Maribon-Montaut «que le 11 courant, il doit être célébré une fête civique par la section des Lombards, laquelle sera terminée par la représentation qu[e] donneront les acteurs du théâtre des sans-culottes, ci-devant Molière, d'une pièce intitulée L'Inauguration de la République française ", c'est-à-dire la sans-culottide de Bouquier, un conventionnel non identifié «observe qu'il est possible que la pièce n'ait pas de succès (36) ». Insuffisance des talents de Bouquier ou lassitude du public à l'endroit de ces pièces de circonstance ? Il reste que l'intervention de ce député montre que les intentions d'une pièce républicaine ne suffisent pas à en assurer le succès et donc que la réalisation des objectifs du gouvernement de « diriger l'esprit public » à partir de pièces de théâtre implique une réception favorable de la part du public. Or ce public ne peut être contrôlé totalement. Pis encore, un accueil défavorable aurait l'effet inverse, c'est-à-dire la dérision du projet lui-même.

Quel a été l'impact de ces décrets sur la vie théâtrale parisienne entre l'hiver 1793 et l'été 1794 ? La période dite de la Terreur et de la «Grande Terreur» a été beaucoup plus celle du Comité de salut public que du Comité d'instruction publique. Outre la censure proprement dite d'une pièce dans son intégralité et la représentation gratuite de pièces républicaines, le contrôle qu'a exercé le Comité de salut public sur la vie théâtrale pendant la période de la Terreur s'exprime aussi par les modifications imposées à certaines répliques d'une pièce afin de la rendre conforme au goût du gouvernement (37). Cette politique culturelle a suscité cependant des

(34) AYOUB et GRENON, CIP, volume IV, Convention, tome 2, p. 464

(35) Id., p. 465.

(36) Archives parlementaires, tome 84, p. 544 (séance du 27 février 1794).

(37) Voir à ce sujet Murielle USANDIVARAS-MII, «Voltaire revu et corrigé par la Révolution française : le cas de La Mort de César», L'Homme et la Nature, 10 (1991), pp. 209-225. On trouvera un court résumé des travaux de cette historienne dans un article intitulé «Le Théâtre de la Révolution française. Étude analytique, historique et socio-critique », Annales historiques de la Révolution française, 1996, $\mathrm{n}^{\circ} 305$, 
protestations au sein du Comité d'instruction publique. Le 17 octobre 1793, Antoine Augustin Renouard, Chardin et Charlemagne fils, signent un mémoire intitulé «observations de quelques patriotes sur la nécessité de conserver les monuments de la littérature et des arts » qui a attiré l'attention du Comité d'instruction publique (38). Ce dernier, partageant les recommandations de ces «patriotes » sur cette «nécessité », a fait imprimer « un plus grand nombre d'exemplaires de cet ouvrage, qui ne peut être trop répandu, vu son utilité pour l'instruction publique » (39).

Après avoir prudemment qualifié de «fort sage » un récent décret de la Convention ordonnant la « destruction de tous les vestiges de la féodalité », ce mémoire effectue cette mise en garde importante : «Mais prenons garde de faire plus que n'a voulu le législateur : il a porté une loi populaire, une loi bienfaisante ; gardons-nous de faire une loi barbare, une loi... destructive des monuments des sciences et des arts » (40). Ces «patriotes », en passant en revue les principaux écrivains français (Fénelon, Racine, Molière, Boileau, La Fontaine, Voltaire) dont l'œuvre sera mutilée, à la fois au niveau du contenu et à celui de la matérialité du livre, dépouillée de tous les ornements royaux, «les fleurs de lis que les relieurs ont presque constamment employées pour ornements », s'insurgent contre ces mesures qualifiées ici de fanatiques : «En vérité, nous ressemblerions un peu trop à ces chrétiens fanatiques qui, dans les premiers siècles de l'Église, détruisaient les précieux ouvrages des Phidias, des Praxitèle, des Protogène, parce qu'ils représentaient des Apollon, des Cybèle, des Jupiter et autres dieux du paganisme » (41). Un décret de la Convention le 24 octobre 1793, défendant de «mutiler ou altérer en aucune manière, sous prétexte de faire disparaître les signes de féodalité ou de royauté, les livres imprimés ou manuscrits », a tranché la question dans le sens voulu par un rapport de Gilbert Romme inspiré des recommandations de Renouard (42). Ce dernier a d'ailleurs réitéré à d'autres occasions, toujours auprès du Comité d'instruction publique, ses arguments en faveur de la conservation de ce patrimoine (43). Mais si l'« habillement» des livres des Corneille, Racine et Voltaire a pu être épargné, la vie théâtrale, elle, subira en 1794 une surveillance encore accrue de la part du Comité de salut public.

pp. 559-560. Voir aussi Roger BARNY, «Le théâtre de Voltaire dans la Révolution française (août 1789 septembre 1793)», $115^{\mathrm{e}}$ congrès national des Sociétés Savantes, Histoire de la Révolution française, Avignon, 1990, pp. 171-198.

(38) AYOUB et GRENON, CIP, volume V, Convention, tome 3, annexe C, pp. 272-274.

(39) Id., p. 274.

(40) Id., p. 272.

(41) Id., p. 273.

(42) Id., p. 276.

(43) Voir notamment une lettre de Renouard datée du 20 octobre 1793 et adressée au Comité d'instruction publique : "Je soutiens que si on veut rhabiller les livres ou placarder les écussons, on gâtera toutes ces raretés si intéressantes ; on mettra la Bibliothèque dans un dérangement et un désordre absolu. » $J d$., p. 275 . 
Un arrêté du Comité de salut public relatif au Théâtre-Français et à l'Opéra daté du 10 mars 1794 (20 ventôse an II), où figurent avec la mention «pour extrait» les noms de Billaud-Varenne, Collot d'Herbois, Robespierre, Couthon, C.-A. Prieur et Carnot, en témoigne éloquemment. À partir d'une délibération sur une pétition qui a été présentée par les sections réunies de Marat, de Mutius Scévola, du Bonnet-Rouge et de L'Unité, le Comité de salut public «arrête » en six articles des mesures qui introduisent une surveillance inédite de la vie théâtrale, tant du point de vue de la censure des pièces, de la propagande patriotique que du contrôle du public. L'article premier annonce ainsi la réouverture du Théâtre de la Nation (l'aile noire de la Comédie-Française) qui « sera uniquement consacré aux représentations données de par et pour le peuple, à certaines époques de chaque mois » (44). Le second article définit l'aspect physique de ce ihéâtre rebaptisé «Théâtre du Peuple», qui sera décoré de «tous les attributs de la liberté» et prévoit la «réquisition» de tous les comédiens des divers théâtres de Paris qui, tour à tour, assureront «les représentations qui devront être données trois fois par décade ». Cet arrêté maintient donc les mesures de propagande prévues par le décret du 2 août 1793 qui initialement devaient se limiter au contexte entourant, pendant un mois, la fête nationale du 10 août 1793.

Mais cet arrêté introduit une mesure sans précédent de contrôle des théâtres : désormais l'accès à la salle du Théâtre du Peuple est réservé uniquement aux spectateurs munis d'une autorisation : « Nul citoyen ne pourra entrer au Théâtre du Peuple s'il n'a une marque particulière qui ne sera donnée qu'aux patriotes » (45). Cet article 3 de l'arrêté du 10 mars 1794 constitue en quelque sorte un aveu de la part du Comité de salut public lui-même que, pour qu'une pièce de théâtre ait les effets prévus, il ne suffit pas que le texte écrit par l'auteur et joué par les acteurs soit patriotique, mais aussi que le public le soit. En d'autres termes, cette censure du public, car comment qualifier autrement une mesure qui prescrit l'obtention d'une autorisation pour assister à une pièce de théâtre, illustre bien l'importance du rôle de coauteur que nous pouvons attribuer au public dans l'élaboration du sens d'une pièce. Enfin, l'arrêté en question charge encore une fois la municipalité de l'exécution de ces mesures inédites de répression, dont la distribution de ces «marques particulières » aux spectateurs (46). Cet arrêté du 10 mars 1794, qui n'a jamais été considéré dans les études portant sur le théâtre révolutionnaire, appuie donc fortement l'argument selon lequel le public constitue un pôle décisif pour appréhender la

(44) AYOUB et GRENON, CIP, volume VI, Convention, tome 4, annexe C, p. 7.

(45) Id.

(46) Id. 
pratique théâtrale (47). Il exprime aussi en quelque sorte le désarroi dans lequel se trouvent à ce moment les membres du Comité de salut public : quelle est en effet la logique d'un théâtre didactique s'adressant à un public déjà converti?

Si le Comité de salut public a donc joué un rôle prépondérant dans le processus d'instrumentalisation et de surveillance des théâtres sous la Terreur, le Comité d'instruction publique a néanmoins été consulté pour des questions relatives à ce que nous pourrions appeler l'aspect qualitatif des pièces de théâtre «données de par et pour le peuple». Un autre arrêté du Comité de salut public, le 6 juin 1794 (18 prairial an II), a chargé expressément la Commission exécutive de l'instruction publique de «tout ce qui concerne la régénération de l'art dramatique et la police des théâtres », ainsi que l'examen des pièces tant anciennes que nouvelles » (48). En exécution de cet arrêté, une commission d'instruction publique a publié le 23 juin 1794 (5 messidor an II) une circulaire intitulée «Commission d'instruction publique. Spectacles » qui, en s'adressant aux directeurs et entrepreneurs de spectacles, aux autorités municipales et aux auteurs dramatiques, devait permettre de «verser dans les spectacles le premier germe de la vie politique ». Les théâtres en effet sont « encore encombrés des débris du dernier régime, de faibles copies de nos grands maîtres, où l'art et le goût n'ont rien à gagner, d'intérêts qui ne nous regardent plus, de moeurs qui ne sont pas les nôtres » (49).

En dépit des velléités de régénération de l'art dramatique exprimées dans cette circulaire signée par le commissaire Payan et son adjoint Fourcade, le procès-verbal du Comité d'instruction publique, le 29 juin 1794, permet de rappeler ce qu'a évoqué Millin de Grandmaison, dès mars 1791, en disant que le patriotisme ne suffit pas à faire le succès d'un ouvrage. Après avoir établi clairement la position de subordination du Comité d'instruction publique par rapport à celui du salut public, ce procèsverbal témoigne des limites esthétiques que rencontrent parfois les pièces patriotiques :

«Un membre fait lecture d'une pièce de vers intitulée Éloge funèbre de Barra. Le Comité entend cette lecture avec un intérêt soutenu. Il reconnaît dans cette production de la verve et un faire poétique. Quelques réflexions sont faites sur la manière moins facile dont est amené le refrain dans quelques strophes; on observe en même temps qu'il serait sans doute peu difficile à

(47) Le débat historiographique sur l'efficacité de la propagande révolutionnaire au théâtre est polarisé d'une manière très nette comme l'évoquent les seuls titres des récents travaux de René TARIN, $L e$ Théâtre de la Constituante ou l'Ecole du peuple, Paris, Honoré Champion, 1998, et de Susan Maslan, «Resisting Representation: Theatre and Democracy in Revolutionary France », Representation, 1995, $\mathrm{n}^{\circ} 52$, pp. $27-51$.

(48) AYOUB et GRENON, CIP, volume VI, Convention, tome 4, annexe B, p. 467.

(49) Id. 
l'auteur de faire disparaître ces imperfections, et par là de rendre la pièce digne de son talent, et de l'objet, et de la République à laquelle ce chant est consacré. » (50)

La réédition de ce procès-verbal offre en annexe un rapport de A.-C. Thibaudeau, daté du $1^{\text {er }}$ juillet 1794 (13 messidor an II), sur la rédaction d'un recueil de pièces patriotiques intitulé Recueil des actions héroüques et civiques des républicains français, où nous retrouvons des critiques beaucoup plus sévères; des critiques qui sont d'autant plus significatives qu'elles proviennent de « réclamations »:

«Il y a deux écueils à éviter. Une suite de faits détachés, isolés, sans aucune liaison, sans chronologie, ne peut pas fixer l'attention, élever l'âme, ni produire de grands effets. Il ne faut pas non plus que les actions héroïques soient noyées dans le luxe des mots, dans des réflexions déplacées, ou des phrases gigantesques, et que l'on voit plus dans le récit l'historien que le héros. » (51)

Puis Thibaudeau propose un véritable livre de recettes à l'usage des propagandistes, visiblement meilleurs patriotes que poètes : «Il est un juste milieu fixé par le goût et par des convenances généralement senties, qui consiste à attacher le cœur et à satisfaire la curiosité. Votre Comité ne s'est pas dissimulé les difficultés de les saisir; mais il s'est efforcé d'en approcher le plus près qu'il lui a été possible » (52).

On constate, le 6 juillet 1794, quelques semaines avant la chute de Robespierre, que la subordination du Comité d'instruction publique au Comité de salut public a considérablement miné les efforts de Payan et Thibaudeau : «Le Comité de salut public a pris des mesures pour que l'on ne jouât sur les théâtres que les pièces capables de propager les principes de la Révolution; en effet, ceci est du ressort du gouvernement : le Comité d'instruction publique n'a pu que lui faire passer toutes celles qui lui sont parvenues » (53). Le Comité d'instruction publique se plaint d'ailleurs du manque de ressources pour mener à bien ses projets et il est bien obligé de reconnaître la suprématie du Comité de salut public : "Mais le Comité d'instruction publique n'a aucun fonds à sa disposition ; il n'a, dans l'organisation actuelle du gouvernement, aucune autorité à exercer dans cette matière; elle appartient tout entière au Comité de salut public, auquel la Convention l'a déléguée » (54).

(50)Id., procès-verbal de la «deux cent soixante-huitième séance. Du 11 messidor an II [29 juin 1794] », p. 465.

(51) Id., annexe A, p. 465.

(52) Id. Voir p. 466 pour le détail des « recettes » proposées.

(Si) Archives parlementaires, tome 92, p. 435 (séance du 6 juillet 1794).

(54)Id. 
Néanmoins, le Comité d'instruction publique a toujours su porter, même dans les circonstances les plus répressives de la «Grande Terreur », quelques semaines avant la chute de Robespierre, un regard critique sur les politiques culturelles de l'an II. La dénonciation la plus éloquente de ce que d'aucuns après Thermidor qualifieront de «vandalisme révolutionnaire» figure dans la Gazette nationale ou Le Moniteur universel du 25 juillet 1794. Elle provient de ces deux membres d'une commission d'instruction publique, Payan et son adjoint Fourcade, dans un rapport non daté, mais qui pourrait bien être antérieur à sa publication dans Le Moniteur (55). J'insisterai sur deux aspects de cette dénonciation : d'abord la date qui, quelle qu'elle soit, précède la chute de Robespierre, ce qui est très significatif puisqu'on a pu dater généralement du lendemain de la réaction thermidorienne uniquement les dénonciations des excès de la Terreur et du terrorisme (56) ; ensuite, l'expression de «l'hébertisme des arts » pour qualifier les tentatives d'instrumentalisation des spectacles (57). Il s'agit d'un texte qui s'intitule : «Rapport sur les corrections de l'opéra de Castor et Pollux [d'après Jean Philippe Rameau]; paroles de Bernard, musique de Candeille ». Le ton de la dénonciation des mutilations qu'a subies cet opéra aux mains des censeurs de cette période est donné dès les premières lignes : «L'ignorance, la grossièreté, la barbarie, enfin tout ce qu'on peut appeler l'hébertisme des arts, marchaient à la contre-révolution par l'abrutissement de la pensée, comme l'hébertisme politique par les complots, le désordre et le meurtre» (58).

En dépit du fait que l'accusation de la faction hébertiste ou «ultra-révolutionnaire », après son arrestation (13 mars 1794), ne soit évidemment pas audacieuse (59), celle de la pratique de la réécriture des pièces de théâtre qui se poursuit après l'exécution d'Hébert (24 mars 1794) mérite d'être soulignée. D'ailleurs, Payan et Fourcade ne parlent pas tant d'Hébert lui-même qui aurait effectué des corrections sur le texte de Castor et Pollux, mais bien

(55) Voir AYOUB et GRENON, CIP, volume VI, Convention, tome 4, p. 470, où est précisé qu'il arrive fréquemment au Moniteur de publier un rapport ou discours avec un retard d'une ou deux décades. Selon Bianchi, (La Révolution culturelle de l'an II, op. cit., p. 225), ce rapport de Payan et Fourcade daterait d'avril 1794.

(56) À telle enseigne que Victor HALLAYS-DABOT, Histoire de la censure théâtrale en France, Genève, Slatkine Reprints, 1970 (Paris, 1862), a cru que le rapport de Payan contenant cette virulente dénonciation est postérieur au 9 thermidor. Ayoub et Grenon ont souligné l'erreur de cet auteur. Voir la «Table générale alphabétique et analytique », fascicule II, M à Z, p. 770.

(57) Albert SOBOUL, Mouvement populaire et gouvernement révolutionnaire en l'an II 1793-1794, Paris, Flammarion, 1973 (1958), a souligné qu'à cette époque de la Grande Terreur « l'accusation d'hébertisme devient le moyen le plus sûr pour perdre des patriotes prononcés ». Payan, d'ailleurs, a été nommé agent national auprès de la Commune de Paris après l'arrestation d'Hébert (13 mars 1794) substitut du procureur Chaumette. Voir p. 398.

(58) La Gazette nationale ou Le Moniteur universel, 307 (vendredi 25 juillet 1794) : 1. Ayoub et Grenon reproduisent également ce rapport dans CIP, volume VI, Convention, tome 4, pp. 470-471.

(59) Surtout de la part de Payan, qui restera en outre jusqu'à la fin fidèle à Robespierre et partagera son sort sur les tréteaux de la guillotine. Voir Claude MANCERON, La Révolution française. Dictionnaire biographique, Paris, Renaudot, 1989, notice sur Claude François Payan, pp. 467-468. 
d'un correcteur, qui n'est pas identifié, «le rival de Bernard », dont le travail, notamment «en supprimant les titres de princes et de rois », a produit des résultats pour le moins incongrus. «Croirait-on que c'est sur le plus beau morceau du poème, sur l'éloge de cette amitié, qui est le ressort de l'action et le nœud de l'intrigue, que le correcteur a dirigé sa faux scythe et vandale?» Payan et Fourcade donnent ensuite l'exemple des vers originaux :

«L'amour te laisse la constance,

Et tu serais la volupté

Si l'homme avait son innocence. »

Transformés en :

«Qui suit les lois avec constance

Ne connait d'autre volupté

Que celle de la bienfaisance. »

L'indignation des auteurs de ce rapport est en effet compréhensible : «Quel style ! quelle incohérence d'idées! Mais correcteur barbare, tu n'as donc pas lu, tu n'as pas du moins entendu celui que tu mutiles !» (60)

Payan et Fourcade retournent ensuite l'intention du correcteur d'honcrer la raison contre lui-même et affirment la souveraineté du « goût et du bon sens » au théâtre : «Les premières lois qu'il faut respecter dans un drame, sont celles du goût et du bon sens; et certes elles auraient dû t'apprendre que tu ne pouvais plus mal servir la raison que de placer là son éloge ». Ces membres de la Commission d'instruction publique concluent leur rapport en généralisant cette critique virulente de «l'hébertisme des arts », qui ne s'applique évidemment pas seulement au cas de Castor et Pollux: «On se rappelle d'ailleurs que le mot de raison ne fut jamais plus vide de sens, que quand certaines gens nous en fatiguèrent les oreilles ». Payan et Fourcade réclament la restitution du texte original (61). Les propos de ces commissaires de l'instruction publique rappellent certainement ceux tenus par Millin de Grandmaison dans La Chronique de Paris, en mars et septembre 1791, qui a critiqué, le premier peut-être, « ces déclamations sans intérêt où l'on répète trois cents fois les mots de citoyens et de liberté ».

Les infortunes de la propagande révolutionnaire au théâtre s'expliquent par la nature de cette pratique culturelle, dont la double publicité, publicité écrite et publicité jouée, lui confère un régime de discours et de configuration du sens particulièrement difficile à contrôler. Le public, mais aussi les acteurs et les actrices, sont des coauteurs de la pièce. À cet égard l'appréhension des comptes rendus de représentations théâtrales est essentielle pour reconstituer historiquement ces moments éphémères. Les

(60) La Gazette nationale ou Le Moniteur universel, 307 (vendredi 25 juillet 1794) : 1.

(61) $I d$ 
critiques de l'art bureaucratisé ne datent pas exclusivement de la période thermidorienne, telle celle de Marie-Joseph Chénier qui sait de quoi il parle, en tant qu'auteur de pièces concernées par le décret du 2 août 1793, à la fois du point de vue de la propagande (Cä̈us Gracchus), que de la censure (Tïmoléon) : «Une commission sage, éclairée, laborieuse, amie de la philosophie, et par conséquent des hommes puisque la philosophie les rend meilleurs, a remplacé cette commission imbécille et conspiratrice qui, sous le joug sanglant de Robespierre, organisoit avec tant de soin l'ignorance et la barbarie ». Le ressentiment de Chénier, caractéristique de la période thermidorienne, n'épargne pas davantage les fêtes nationales, «ces processions éternelles, qui consument une journée entière, qui fatiguent le peuple sans l'amuser » (62). Mais la censure et la propagande révolutionnaire au théâtre ont sévi sur un public qui, même sous la Terreur, n'a guère été médusé.

\author{
Martin NADEAU \\ Université McGill \\ 5534 8e avenue, Montréal (Québec) \\ Canada \\ HIY 2L4 \\ mnadeauparis@hotmail.com.
}

(62) Archives parlementaires, tome 99, pp. 259-260 (séance du 18 octobre 1794). Voir à ce sujet Bronislaw BACZKO, Comment sortir de la Terreur. Thermidor et la Révolution, Paris, Gallimard, 1989. 